\title{
Heavy Hadron Spectroscopy at CDF
}

\author{
Juan Pablo Fernández Ramos \\ CIEMAT, Av. Complutense 22, E-28040 Madrid, Spain \\ On behalf of the CDF Collaboration
}

\begin{abstract}
We present recent CDF results on the properties of hadrons containing heavy quarks. These include measurements of charm and $\Sigma_{b}^{-} \Sigma_{b}^{*-}$ baryon's masses, lifetimes and masses of $\Omega_{b}^{-}$, $\Xi_{b}^{-}$and $B_{c}^{-}$and a measurement of exclusive $B^{+}, B^{0}$ and $\Lambda_{b}$ lifetimes as well as lifetime ratios (charge conjugate modes are implied throughout the text). We also summarize new measurements of exotic particles $X(3872)$ and $Y(4140)$.
\end{abstract}

Keywords: Elementary Particles, High Enery Physics, Heavy Hadron Spectroscopy, CDF

PACS: $14.20 . \mathrm{Mr}, 14.40 . \mathrm{Nd}$

\section{INTRODUCTION}

The interaction between a $b$ quark and the other quark(s) in a $B$ hadron is based on the strong interaction or Quantum Chromodynamics (QCD). It is often stated that heavy quark hadrons are the hydrogen atom of QCD since we can use perturbative QCD to describe the potential quite well. $\mathrm{B}$ hadrons probe a unique region of parameter space (i.e., mass, energy, momentum, velocity) that can be studied using a wide range of tools like potential models, HQET [1,2] and lattice gauge calculations. Besides, measurements of $B$ hadron lifetimes study the interplay between the strong and weak interaction and test the validity of HQE $[3,4]$ and the lifetime-hierarchy of the $B$ hadrons. The HQE technique is also used to supply input for the extracion of elements of the Cabibbo-Kobayashi-Maskawa(CKM) quark mixing matrix [5, 6]. Furthermore, because of its rich mass spectrum and the relatively narrow widths of the resonances, the charmed baryon system makes a good testing ground for the heavy quark symmetry.

The Fermilab Tevatron Collider is currently the most copious source of $B$ hadrons, thanks to the large $b \bar{b}$ production cross-section in $1.96 \mathrm{TeV} p \bar{p}$ collisions. With the current statistics, more than $7 \mathrm{fb}^{-1}$ on tape $\left(10 \mathrm{fb}^{-1}\right.$ on tape by the end of 2011), the Tevatron $B$ Physics program allows for a wide range of measurements that are competitive or complementary with $B$-factories. We summarize here some of the recent experimental progress in the measurements related to $b$ hadron spectroscopy. We will touch not only masses but also quantum numbers and lifetimes in this paper.

\section{THE CDF II DETECTOR}

The elements of the CDF II detector most relevant for $b$ Physics analysis are the tracker, the particle identification detectors and the muon system. The CDF tracker is located within a $14.1 \mathrm{kG}$ solenoidal magnetic field and it is composed of silicon detectors [7] surrounded by a drift chamber, COT [8]. The achieved performance of the integrated 
CDF tracker is a transverse momentum resolution $\sigma\left(p_{t}\right) / p_{t}^{2}=0.15 \%(\mathrm{GeV} / \mathrm{c})^{-1}$ and an impact parameter resolution $\sigma(d)=35 \mu \mathrm{m} @ 2 \mathrm{GeV} / \mathrm{c}$. The CDF central muon detector [9] is located around the outside of the central calorimeter, which is 5.5 interaction lengths thick, at a radius of $347 \mathrm{~cm}$ from the beam axis. The pseudorapidity coverage of the muon detector is $|\eta|<1$.

\section{THE CDF TRIGGER}

The $b \bar{b}$ production cross section of $\approx 40 \mu b$ [10] is large compared to $B$-factories. However, the Tevatron cross section is orders of magnitude smaller than the total inelastic cross section of $\approx 50 \mathrm{mb}$. The CDF experiment triggers on final states containing single or di-leptons to select high statistics samples of b-hadron decays. Semileptonic $B \rightarrow l v_{l} X$ plus charmonium $B \rightarrow J / \psi X \rightarrow\left[l^{+} l^{-}\right] X$ decays are of the order of $20 \%$ of the $B$ meson widths. In addition $\mathrm{CDF}$ has a special trigger (hadronic trigger) to select events based upon track impact parameter (the minimum distance between the track and the beam), called SVT [11]. It is basically a device able to reconstruct tracks in silicon, with offline-like resolution in the $20 \mu$ s allowed by trigger latency, and select events containing tracks originated in a vertex displaced from the primary. These events are enriched of heavy flavor contents, thanks to the higher mean-valued lifetimes of $B$ hadrons.

\section{THE PHYSICS PROGRAM ON HEAVY QUARK HADRON SPECTROSCOPY}

\section{Measurement of the resonance properties of charm baryons}

By means of a proper inclusion of kinematical threshold effects in the resonant decays $\Lambda_{c}(2595)^{+} \rightarrow \Sigma_{c}(2455)^{0,++} \pi^{+,-}$, a direct experimental determination of the pion coupling constant $\mathrm{h} 2$ in the chiral Lagrangian is feasible [12]. The knowledge of $\mathrm{h} 2$ provides information about other excited charm and bottom baryons $[13,14]$.

A measurement of the resonance properties of $\Lambda_{c}(2595)^{+}$and $\Lambda_{c}(2625)^{+}$in its decays to $\Lambda_{c}^{+} \pi^{+} \pi^{-}$as well as $\Sigma_{c}(2455)^{++, 0}$ and $\Sigma_{c}(2520)^{++, 0}$ in its decays to $\Lambda_{c}^{+} \pi^{ \pm}$is reported on [15]. The measurement is performed using $5.2 \mathrm{fb}^{-1}$ of integrated luminosity. Exploiting the largest available sample in the world, we measure masses and widths which are competitive to the world averages for $\Sigma_{c}$ states and significantly more precise than the world averages for excited $\Lambda_{c}$ states. It turns out that the $\Lambda_{c}(2595)^{+}$mass shape is affected by kinematical threshold effects in the resonant subdecays $\Lambda_{c}(2595)^{+} \rightarrow$ $\Sigma_{c}(2455)^{++, 0} \pi$. This leads to a $\Lambda_{c}(2595)^{+}$mass which is approximately $3 \mathrm{MeV} / \mathrm{c}^{2}$ lower than the previously measured values [16]. The whole set of masses results follows : $\mathrm{m}\left(\Sigma_{c}(2455)^{++}\right)=2453.90 \pm 0.13(\exp ) \pm 0.14(\mathrm{PDG}) \mathrm{MeV} / \mathrm{c}^{2}, \mathrm{~m}\left(\Sigma_{c}(2455)^{0}\right)=$ $2453.74 \pm 0.12 \pm 0.14 \mathrm{MeV} / \mathrm{c}^{2}, \mathrm{~m}\left(\Sigma_{c}(2520)^{++}\right)=2517.19 \pm 0.46 \pm 0.14 \mathrm{MeV} / \mathrm{c}^{2}$, $\mathrm{m}\left(\Sigma_{c}(2520)^{0}\right)=2519.34 \pm 0.58 \pm 0.14 \mathrm{MeV} / \mathrm{c}^{2}, \mathrm{~m}\left(\Lambda_{c}(2595)^{+}\right)=2592.25 \pm 0.24 \pm$ $0.14 \mathrm{MeV} / \mathrm{c}^{2}$ and $\mathrm{m}\left(\Lambda_{c}(2625)^{+}\right)=2628.11 \pm 0.13 \pm 0.14 \mathrm{MeV} / \mathrm{c}^{2}$. 
The Figure 1 (a) shows the $\Lambda_{c}(2595)^{+}$and $\Lambda_{c}(2625)^{+}$Q-value distribution together with the mass fits.
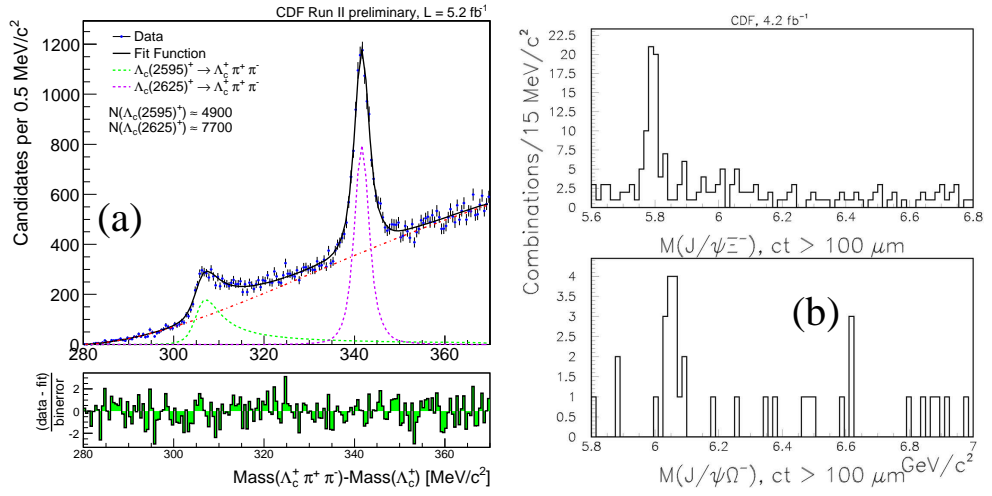

FIGURE 1. (a) $\Lambda_{c}(2595)^{+}$and $\Lambda_{c}(2625)^{+}$Q-value distribution (b) $J / \psi \Xi^{-}$(top) and $J / \psi \Omega^{-}$(bottom) invariant masses .

\section{Measurement of the Bottom Baryon Resonances $\Sigma_{b}^{-} \Sigma_{b}^{*-}$}

CDF has recently published [17] the best measurements of the heavy baryons $\Sigma_{b}^{-}$and $\Sigma_{b}^{*-}$ in decays to $\Lambda_{b} \pi_{\text {soft }}^{-}$. This study follows the observation of these states published in [18]. The new analysis is based on a data sample corresponding to an integrated luminosity of $6.0 \mathrm{fb}^{-1}$.

Predictions for the $\Sigma_{b}^{-} \Sigma_{b}^{*-}$ masses come from non-relativistic and relativistic potential quark models, $1 / N_{c}$ expansion, quark models in the HQET approximation, sum rules, and lattice quantum chromodinamics calculations. On the basis of those predictions, we expect $\mathrm{m}\left(\Sigma_{b}\right)-\mathrm{m}\left(\Lambda_{b}\right) \simeq 180-210 \mathrm{MeV} / \mathrm{c}^{2}, \mathrm{~m}\left(\Sigma_{b}^{*}\right)-\mathrm{m}\left(\Sigma_{b}\right) \simeq 10-40$ $\mathrm{MeV} / \mathrm{c}^{2}$ and $\mathrm{m}\left(\Sigma_{b}^{(*)-}\right)-\mathrm{m}\left(\Sigma_{b}^{(*)+}\right) \simeq 5-7 \mathrm{MeV} / \mathrm{c}^{2} \quad[19,20,21,22]$. The difference between the isospin mass splittings of the $\Sigma_{b}^{*}$ and $\Sigma_{b}$ multiplets is predicted to be $\left[m\left(\Sigma_{b}^{*+}\right)-m\left(\Sigma_{b}^{*-}\right)\right]-\left[m\left(\Sigma_{b}^{+}\right)-m\left(\Sigma_{b}^{-}\right)\right] \simeq 0.4 \pm 0.07 \mathrm{MeV} / \mathrm{c}^{2}[23,24]$. The natural width of $\Sigma_{b}, \Sigma_{b}^{*}$ bayrons is expected to be dominated by the P-wave one pion transition $\Sigma_{b} \rightarrow \Lambda_{b} \pi$, whose partial width depends on the available phase space and the pion coupling to a constituent quark. Using an HQET prediction [25], the natural widths for the expected $\Sigma_{b} \Sigma_{b}^{*}$ masses are $\Gamma\left(\Sigma_{b}^{ \pm}\right) \simeq 7 \mathrm{MeV} / \mathrm{c}^{2}$ and $\Gamma\left(\Sigma_{b}^{(*) \pm}\right) \simeq 13 \mathrm{MeV} / \mathrm{c}^{2}$.

The four $\Lambda_{b} \pi_{\text {soft }}^{-}$resonant states masses are measured to be: $\mathrm{m}\left(\Sigma_{b}^{-}\right)=5815.5_{-0.5}^{+0.6}$ (stat) \pm 1.7 (syst) $\mathrm{MeV} / \mathrm{c}^{2}, \mathrm{~m}\left(\Sigma_{b}^{+}\right)=5811.2_{-0.8}^{+0.9}$ (stat) \pm 1.7 (syst) $\mathrm{MeV} / \mathrm{c}^{2}, \mathrm{~m}\left(\Sigma_{b}^{*-}\right.$ ) $=5835.0 \pm 0.6$ (stat) \pm 1.8 (syst) $\mathrm{MeV} / \mathrm{c}^{2}, \mathrm{~m}\left(\Sigma_{b}^{*+}\right)=5832.0 \pm 0.7$ (stat) \pm 1.8 (syst) $\mathrm{MeV} / \mathrm{c}^{2}$ in line with the expectations from above [19, 20, 21, 22].

We report on the first measurement of isospin mass splittings for $J^{P}=\frac{1}{2}^{+}$and $J^{P}=\frac{3}{2}^{+}$ isospin multiplets of $\Sigma_{b}^{*}$ bottom baryons: $\mathrm{m}\left(\Sigma_{b}^{+}\right)-\mathrm{m}\left(\Sigma_{b}^{-}\right)=-4.2_{-0.9}^{+1.1}$ (stat) ${ }_{-0.09}^{+0.07}$ (syst) $\mathrm{MeV} / \mathrm{c}^{2}, \mathrm{~m}\left(\Sigma_{b}^{*+}\right)-\mathrm{m}\left(\Sigma_{b}^{*-}\right)=-3.0 \pm 0.9$ (stat) ${ }_{-0.13}^{+0.12}$ (syst) $\mathrm{MeV} / \mathrm{c}^{2}$. The difference between the isospin mass splittings is in agreement with above expectations $[23,24]$. 
We also report on the first measurement of the widths of these states: $\Gamma\left(\Sigma_{b}^{-}\right)=4.3_{-2.1}^{+3.1}$ (stat) ${ }_{-1.1}^{+1.0}$ (syst) $\mathrm{MeV} / \mathrm{c}^{2} \Gamma\left(\Sigma_{b}^{+}\right)=9.2_{-2.9}^{+3.8}$ (stat) ${ }_{-1.1}^{+1.0}$ (syst) $\mathrm{MeV} / \mathrm{c}^{2} \Gamma\left(\Sigma_{b}^{*-}\right)=6.4_{-1.8}^{+2.2}$ (stat) ${ }_{-1.1}^{+0.7}$ (syst) $\mathrm{MeV} / \mathrm{c}^{2} \Gamma\left(\Sigma_{b}^{*+}\right)=10.4_{-2.2}^{+2.7}$ (stat) ${ }_{-1.2}^{+0.8}$ (syst) $\mathrm{MeV} / \mathrm{c}^{2}$.

The Figure 2 shows the $\Sigma_{b}^{-}, \Sigma_{b}^{*-}$ (a) and $\Sigma_{b}^{+}, \Sigma_{b}^{*+}$ (b) Q-value distribution together with their fits.
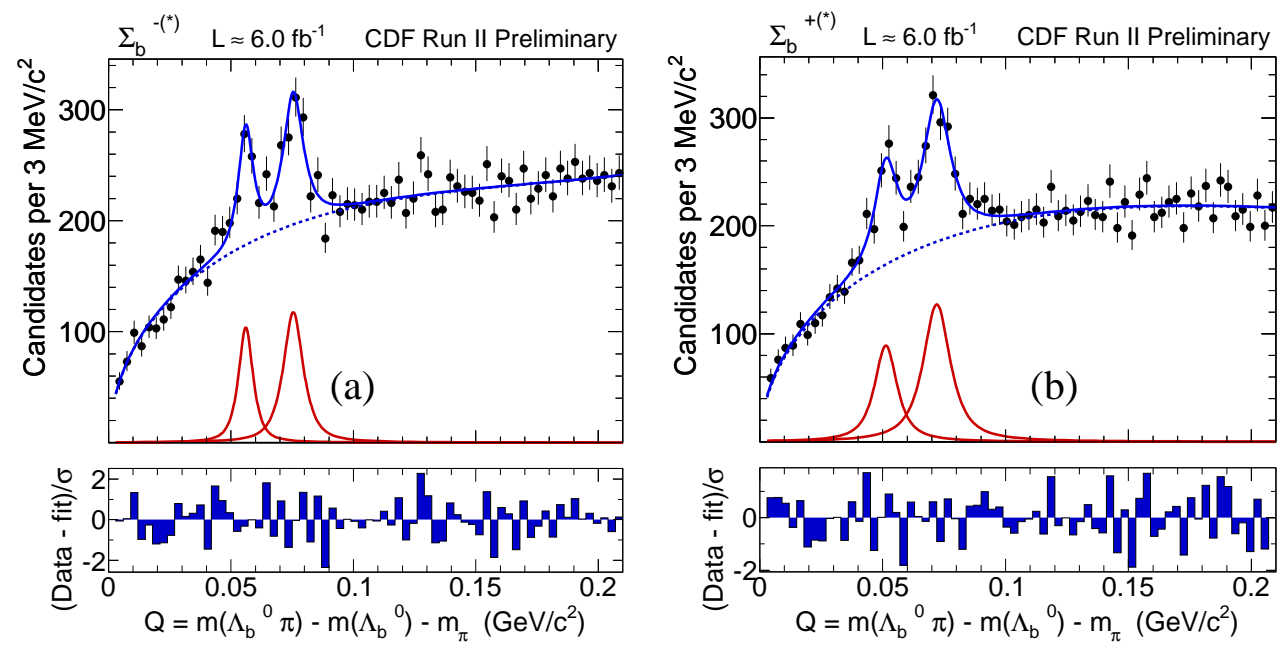

FIGURE 2. (a) $\Sigma_{b}^{-}$, $\Sigma_{b}^{*-} \mathrm{Q}$-value distribution and (b) $\Sigma_{b}^{+}$, $\Sigma_{b}^{*+} \mathrm{Q}$-value distribution for $\Lambda_{b} \pi_{\text {soft }}^{-}$candidates. Unbinned fits are also shown.

\section{$\Omega_{b}^{-}, \Xi_{b}^{-}$and $B_{c}^{-}$mass and lifetime}

CDF has recently published [26] the best mass measurements of $\Omega_{b}^{-}$and $\Xi_{b}^{-}$as well as $\Omega_{b}^{-}$and $\Xi_{b}^{-}$lifetimes. This is the first lifetime measurement of $\Omega_{b}^{-}$ever. This paper reports the observation of the bottom, doubly-strange baryon $\Omega_{b}^{-}$through the decay chain $\Omega_{b}^{-} \rightarrow J / \psi \Omega^{-}$, where $J / \psi \rightarrow \mu^{+} \mu^{-}, \Omega^{-} \rightarrow \Lambda K^{-}$, and $\Lambda \rightarrow p \pi^{-}$, using $4.2 \mathrm{fb}^{-1}$ of data. A signal is observed whose probability of arising from a background fluctuation is $4.0 \times 10^{-8}$, or 5.5 Gaussian standard deviations. The Figure 1 (b) shows the $J / \psi \Xi^{-}$ and $J / \psi \Omega^{-}$invariant masses. The $\Omega_{b}^{-}$mass is measured to be $6054.4 \pm 6.8$ (stat.) \pm 0.9 (syst.) $\mathrm{MeV} / c^{2}$. The lifetime of the $\Omega_{b}^{-}$baryon is measured to be $1.13_{-0.40}^{+0.53}$ (stat.) \pm 0.02 (syst.) ps. In addition, for the $\Xi_{b}^{-}$baryon we measure a mass of $5790.9 \pm 2.6$ (stat.) \pm 0.8 (syst.) $\mathrm{MeV} / \mathrm{c}^{2}$ and a lifetime of $1.56_{-0.25}^{+0.27}$ (stat.) \pm 0.02 (syst.) ps. The already small experimental uncertainties start to challenge the theoretical models [27, 28, 29].

The $B_{c}^{-}$mass measurement with $\left(2.4 \mathrm{fb}^{-1}\right)$ is presented in [30]. A search is made for the exclusive decay $B_{c}^{-} \rightarrow J / \Psi \pi^{-}$. This analysis emphasizes the removal of background by tuning selection requirements on the $B^{-} \rightarrow J / \psi K^{-}$signal and sideband background regions using the data. The selection requirements are fixed before looking at the $M\left(J / \psi \pi^{-}\right)$distribution for $B_{c}^{-}$. A signal of $108 \pm 15$ candidates is observed, with a significance that exceeds $8 \sigma$. The mass of the $B_{c}^{-}$meson is measured to be $6275.6 \pm 2.9 \pm 2.5 \mathrm{MeV} / \mathrm{c}^{2}$ in good agreement with theory [31]. 
The $B_{c}^{-}$lifetime in $1 \mathrm{fb}^{-1}$ of inclusive $J / \psi \rightarrow \mu^{+} \mu^{-}$data is measured using $B_{c}^{-} \rightarrow$ $J / \psi+l^{-}+X$ decays [32], where $l^{-}$can be an electron or a muon and $X$ are unmeasured particles. We analyze $J / \psi+l^{-}$candidates with an invariant mass between 4.0 and $6.0 \mathrm{GeV} / \mathrm{c}^{2}$, which is the range in which we expect the $B_{c}^{-}$signal to lie. A variety of backgrounds are present in the $J / \psi+l^{-}$signal mass window and the contribution of these backgrounds to the lifetime is estimated using both data and Monte Carlo simulation. An extended log likelihood fit of the $J / \psi+l^{-}$lifetime is performed and an average lifetime of the $B_{c}^{-}$meson of $c \tau=142.5_{-14.8}^{+15.8}$ (stat.) \pm 5.5 (syst.) microns is found.

\section{$B^{+}, B^{0}$ and $\Lambda_{b}$ lifetimes and ratios}

The lifetime of a ground-state hadron containing a $b$ quark and lighter quarks is largely determined by the charged weak decay of the $b$ quark. Interactions involving the lighter quarks, referred to as non-spectator interactions, alter $b$-hadron lifetimes at approximately the $10 \%$ level. The ratios of $b$-hadron lifetimes are predicted by the Heavy Quark Expansion (HQE) [33, 34, 35, 36]. This framework of theoretical calculation is used to predict low energy QCD effects in many flavor observables, some of which are critical to high profile new physics searches. For example, the standard model expectations of the width difference in the $B_{s}^{0}$ system and the semileptonic asymmetry are calculated using the HQE. The measurement of lifetime ratios provides a relatively simple and accurate way to test the HQE framework since no new physics effects are expected to be visible in lifetimes.

A measurement of the lifetimes of the $B^{+}, B^{0}$ and $\Lambda_{b}$ hadrons and of the lifetime ratios $\tau_{B^{+}} / \tau_{B^{0}}$, and $\tau_{\Lambda_{b}} / \tau_{B^{0}}$ is presented in [37]. The measurement is performed using exclusive decays to states containing a $J / \psi$ with $J / \psi \rightarrow \mu^{+} \mu^{-}$. The work reported here is based on an integrated luminosity of $4.3 \mathrm{fb}^{-1}$.

The goal of this set of measurements is to measure lifetimes as precisely as possible and in a consistent way across all of the decay channels considered. Systematic errors are controlled to the level necessitated by the $B^{0}$ and $B^{+}$modes, and then we apply the same methods across the board to $\Lambda_{b}$. The vertex formed by the two tracks from the $J / \psi$ is used as an estimate of the transverse decay length (distance from primary to secondary $J / \psi$ vertex projected into the transverse momentum of the $J / \psi$ candidate) so that systematic errors common to the estimate of decay length cancel to some extent in the ratio of lifetimes.

The lifetimes are extracted using an unbinned maximum likelihood method. The likelihood function $\mathscr{L}$ is multivariate, and is based on the probability of observing a candidate event with $m$ (mass), $c t$ (proper decay time), $\sigma^{c t}$ (proper decay time's error) and $\sigma^{m}$ (mass' error). The likelihood is factorized in normalized probability density functions (PDF) for those variables and is a sum of signal plus background PDF products. The components the take part in each PDF are described in [37].

The same resolution model, $\mathscr{R}$, is used for signal and background events. It is determined from the data in the mass sidebands, where between $80-90 \%$ of the background events are expected to originate from the primary vertex, depending on decay channel and model. $\mathscr{R}$ is modeled as three Gaussians centered at $\mathrm{t}=0$; the symmetry of this model 
is motivated by simulation, while the number of components is motivated by the data, specifically, a study of resolution in an inclusive sample of $\mathrm{J} / \psi$ events. The width of the Gaussians is given by the candidate measured uncertainty, $\sigma^{c t}$, multiplied by a scale factor, where the scale factor is allowed to dependent on $\sigma^{c t}$. The overall systematic reduction for the analysis by using the above resolution model is for example from 0.016 ps to 0.008 ps (for $B^{0}$ ). The systematic error is limited by detector alignment (that cancel in ratios). The alignment was determined in $\mathrm{MC}$ retracking with different alignment constants, taking the largest shift $(2 \mu \mathrm{m})$. For lifetime ratios, the total uncertainty has larger contributions from systematic uncertainties due to resolution and mass models.

Various projections of the likelihood function are compared with the data in Fig. 3.
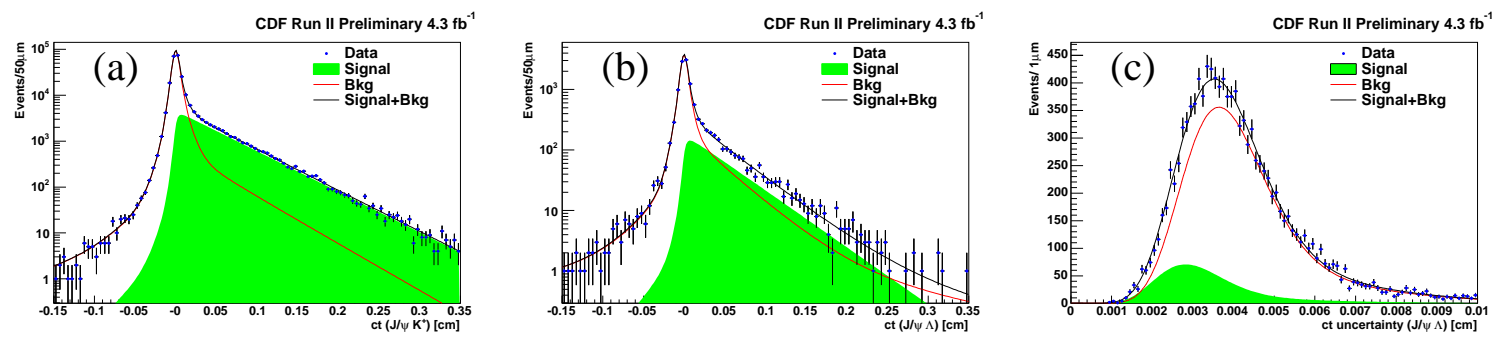

FIGURE 3. (a) $c t$ fit projection for $B^{+} \rightarrow J / \psi K^{+}$candidates, (b) $c t$ fit projection and (c) $\sigma^{c t}$ fit projection for $\Lambda_{b}^{0} \rightarrow J / \psi \Lambda^{0}$ candidates.

We measure $\tau_{B^{+}}=1.639 \pm 0.009 \pm 0.009 \mathrm{ps}, \tau_{B^{0}}=1.507 \pm 0.010 \pm 0.008 \mathrm{ps}$ where the two $B^{0}$ measurements have been combined, and the first uncertainty is statistical, and the second systematic. These results are consistent and improve upon the leading measurements from Belle [38]. The similarities between the decay channels allow for the accurate determination of the ratio $\tau_{B^{+}} / \tau_{B^{0}}=1.088 \pm 0.009$ (stat) \pm 0.004 (syst) which favors a slightly higher value than the current average of $1.071 \pm 0.009$ [39]. These results are consistent with the current HQE predictions [40], giving further confidence in this theoretical framework, and also provide an accurate test for future Lattice QCD calculations. For the $\Lambda_{b}^{0}$ we measure $\tau_{\Lambda_{b}^{0}}=1.537 \pm 0.045 \pm 0.014$ ps and $\tau_{\Lambda_{b}^{0}} / \tau_{B^{0}}=$ $1.020 \pm 0.030 \pm 0.008$. This measurement is the most precise measurement of $\tau_{\Lambda_{b}}$ and is consistent with the previous measurement in this decay channel of $\tau_{\Lambda_{b}^{0}}=1.593_{-0.078}^{+0.083} \pm$ $0.033 \mathrm{ps}$ [41] but is more than $2 \sigma$ above the world average of $1.389_{-0.048}^{+0.049}$ ps. The ratio is also higher than the predicted values of $0.88 \pm 0.05$ [42] but in agreement with 1.063 \pm 0.027 [43]. Note that the complete NLO-QCD calculations have not been included in any of the theoretical results. Nevertheless, improvements both from the experimental and theoretical sides will be necessary before $\tau_{\Lambda_{b}}$ is well understood.

\section{Exotic states}

Since $X(3872)$ in 2003 [44] many exotic mesons have been found. QCD predictions for these new states are: multiquark mesons molecule $\left(q q^{6} q q^{6}\right)$, hybrid mesons $(q g q)$ and glueball $(\mathrm{gg})$ [45]. We report on properties of $X(3872)$ as well as an update on the mass measurement together with the exotic particle $Y(4140)$. 
Analysis of the quantum numbers JPC of the X(3872) and precision Measurement of its mass. An analysis of the angular distributions and correlations together with the mass of the $X(3872)$ reconstructed via its decay to $J / \psi \pi^{+} \pi^{-}$using $780 \mathrm{pb}^{-1}\left(2.4 \mathrm{fb}^{-1}\right.$ for the mass measurement) of integrated luminosity is presented in $[46,47]$.

Constraints on spin, parity, and charge conjugation parity of the $X(3872)$ are derived by comparing measured angular distributions of the decay products with predictions for different $J^{P C}$ hypotheses. The assignments $J^{P C}=1^{++}$and $2^{-+}$are the only ones consistent with the data.

Furthermore, the possible existence of two nearby mass states is investigated in [47]. Within the limits of the experimental resolution the data are consistent with a single state, and having no evidence for two states an upper limits on the mass difference between two hypothetical states is set. Assuming each state contributes equally to the observed peak, the $95 \%$ confidence level upper limit on the mass difference is $3.6 \mathrm{MeV} / \mathrm{c}^{2}$. Under the single-state model the $X(3872)$ mass is measured to be $3871.61 \pm 0.16$ (stat) \pm 0.19 (syst) $\mathrm{MeV} / \mathrm{c}^{2}$, which is the most precise determination to date.

Observation of a Narrow Near-Threshold Structure in the $J / \psi \phi$ Mass Spectrum in $B^{+} \rightarrow J / \psi \phi K^{+}$Decays . Observation is reported [48] for a structure near the $J / \psi \phi$ threshold, in $B^{+} \rightarrow J / \psi \phi K^{+}$decays with a statistical significance of beyond 5 standard deviations. There are $19 \pm 6$ events observed for this structure at a mass of $4143.4_{-3.0}^{+2.9}$ (stat) \pm 0.6 (syst) $\mathrm{MeV} / \mathrm{c}^{2}$ and a width of $15.3_{-6.1}^{+10.4} \pm 2.5 \mathrm{MeV} / \mathrm{c}^{2}$, which are consistent with the previous measurements reported as evidence of the Y(4140). A new structure at mass of roughly $4270 \mathrm{MeV} / \mathrm{c}^{2}$ with approximately $3-\sigma$ is also reported in the above refence.

\section{CONCLUSIONS}

In this paper we have reviewed $\mathrm{CDF}$ results concerning properties of heavy quark hadrons. The CDF collaboration is very active in this area. The broad physics program includes mass measurements of charm baryons and $\Sigma_{b}^{-} \Sigma_{b}^{*-}$ states, mass and lifetime measurements of $\Omega_{b}^{-}$and $\Xi_{b}^{-}$baryons and of $B_{c}^{-}$meson, lifetime measurements of $B^{+}$, $B^{0}$ and $\Lambda_{b}$ and finnaly establishment and properties of the $X(3872)$ and $Y(4140)$ exotic particles. We expect more results from the Tevatron which will accumulate more data until the end of Run II currently scheduled to conclude in 2011.

\section{ACKNOWLEDGMENTS}

I would like to thank the organizers of the 2010 QCD Workshop for the opportunity to speak at this Conference and the colleagues from the CDF Collaboration for assisting me while preparing talk and document. 


\section{REFERENCES}

1. E. Eichten, Nucl. Phys. Proc. Suppl. 4, 170 (1988).

2. E. Eichten, and B. Hill, Phys. Lett. B 234, 511 (1990).

3. M. Neubert, Adv. Ser. Dir. High Energy Phys. 15, 239 (1998).

4. I. Bigi-et al., Annu. Rev. Nucl. Part. Sci. 47, 591 (1997).

5. N. Cabibbo, Phys. Rev. Lett. 10, 531 (1963).

6. M. Kobayashi, and T. Maskawa, Prog. Theor. Phys. 49, 652 (1973).

7. A. Sill-et al., Nucl. Instrum. Meth. A 530, 1 (2004).

8. T. Affolder-et al., Nucl. Instrum. Meth. A 526, 249 (2004).

9. G. Ascoli-et al., Nucl. Instrum. Meth. A 268, 33 (1988).

10. D. Acosta-et al., Phys. Rev. D 71, 032001 (2005).

11. W. Ashmanskas-et al, Nucl. Instrum. Meth. A 518, 532 (2004).

12. A. Blechman-et al, Phys. Rev. D 67, 070433 (2003).

13. D. Pirjol-et al, Phys. Rev. D 56, 5483 (1997).

14. G. Chiladze, and A. Falk, Phys. Rev. D 56, 6738 (1997).

15. [to be] submitted to Phys. Rev. Lett. (2010), URL http://www-cdf. fnal.gov/physics/ new/bottom/100701.blessed-Charm_Baryons/.

16. K. Edwards-et al, Phys. Rev. Lett. 74, 3331 (1995).

17. [to be] submitted to Phys. Rev. Lett. (2010), URL http://www-cdf.fnal.gov/physics/ new/bottom/100701.blessed-Charm_Baryons/.

18. T. Aaltonen-et al, Phys. Rev. Lett. 99, 202001 (2007).

19. C. Albertus-et al, Nucl. Phys. A 740, 333 (2004).

20. D. Ebert-et al, Phys. Rev. D 72, 034026 (2005).

21. R. Roncaglia-et al, Phys. Rev. D 52, 1722 (1995).

22. M. Karliner-et al, Phys. Lett. B 575, 249 (2003).

23. J. Rosner-et al, Phys. Rev. D 57, 4310 (1998).

24. J. Rosner-et al, Phys. Rev. D 75, 013009 (2007).

25. J. Körner-et al, Prog. Part. Nucl. Phys. 33, 787 (1994).

26. T. Aaltonen-et al, Phys. Rev. Lett. 80, 072003 (2009).

27. E. Jenkins-et al, Phys. Rev. D 77, 034012 (2008).

28. M. Karliner-et al, Ann. Phys. 324, 2 (2009).

29. T. Lewis-et al, Phys. Rev. D 79, 014502 (2009).

30. T. Aaltonen-et al, Phys. Rev. Lett. 100, 182002 (2008).

31. I. Alison-et al, Phys. Rev. Lett. 94, 172001 (2005).

32. not to be publised (2008), URL http://www-cdf.fnal.gov/physics/new/bottom/ 080327.blessed-BC_LT_SemiLeptonic/.

33. M. Beneke-et al, Nucl. Phys. B639, 389 (2002).

34. E. Franco-et al, Nucl. Phys. B633, 212 (2002).

35. C. Tarantino-et al, Eur. Phys. J. c33, S895 (2004).

36. A. j. Lenz-et al, AIP Conf. Proc. 1026, 36 (2008).

37. [to be] submitted to Phys. Rev. Lett. (2010), URL http://www-cdf. fnal.gov/physics/ new/bottom/091217.blessed-JpsiX4.3/jpsix.html.

38. K. Abe-et al, Phys. Rev. D 71, 072003 (2005).

39. C. Amsler-et al, Phys. Lett. B 667, 1 (2008).

40. C. Amsler-et al, Phys. Lett. B 667, 20068 (2008).

41. A. Abulencia-et al, Phys. Rev. Lett. 98, 122001 (2007).

42. I. Bigi-et al, arXiv:0310241 [hep-ph] (2003).

43. A. Lenz-et al, arXiv:0802.0977 [hep-ph] (2008).

44. S.-K. Choi-et al, Phys. Rev. Lett. 91, 262001 (2003).

45. S. Godfrey, and S. Olsen, Annu. Rev. Nucl. Part. Sci. 58, 73 (2008).

46. A. Abulencia-et al, Phys. Rev. Lett. 98, 132002 (2007).

47. T. Aaltonen-et al, Phys. Rev. Lett. 103, 152001 (2009).

48. T. Aaltonen-et al, Phys. Rev. Lett. 102, 242002 (2009). 